\title{
Rechtsgefühl, Verrechtlichung und Wandel des Rechts
}

Gewidmet dem Andenken meines Vaters, Dr. jur. Joseph Kaufmann (1884-1970), dem Anwalt, Richter und Politiker, anläßlich seines 100 . Geburtstages.

Das Rechtsgefühl ist nichts in der Wirklichkeit eindeutig Gegebenes, sondern ein Konstrukt von Juristen. Für eine Vertiefung der Rede vom Rechtsgefühl muß man sich zunächst ihrer Funktion in juristischen Diskursen vergewissern. Psychologische, sozialpsychologische oder soziologische Interpretationen sind nur insoweit von Belang, als sie sich auf diese Funktion beziehen lassen.

In juristischen Diskursen ist vom Rechtsgefühl mit Bezug auf zwei unterschiedliche Sozialkontexte die Rede: Es wird einerseits als spezifische, erworbene Qualität von Juristen, insbesondere in ihrer richterlichen Funktion, bestimmt - etwa als Sinn für die richtige Anwendung oder Fortentwicklung des Rechts, als gerechtes Urteilsvermögen oder als Fähigkeit zum Judiz. Es wird andererseits als Inbegriff von Dispositionen und Fähigkeiten der juristischen Laien im Verhältnis zum Recht bestimmt, etwa als Rechtswissen, Rechtsgefühl im engeren Sinne und Rechtswillen (Riezler, Kriele) oder als (Un)Rechtsbewußtsein, Rechtssicherheitsgefühl und Gerechtigkeitsglaube (vgl. FN 4).

Der Begriff des Rechtsgefühls (in beiden Sozialkontexten) bezieht sich sodann auf zwei unterschiedliche Problemkontexte: Zunächst auf Probleme der Rechtssetzung; auch wenn das Rechtsgefühl heute übereinstimmend nicht mehr als Rechtsquelle akzeptiert wird, so erscheint es doch als Bedingung für eine angemessene Vermittlung zwischen den immanenten Konsistenzbedingungen einer gegebenen Rechtsordnung und der Dynamik regelungsbedürftiger sozialer Sachverhalte im Prozeß der Rechtssetzung und Rechtsfortbildung unverzichtbar. Darüber hinaus taucht der Begriff des Rechtsgefühls jedoch auch im Kontext der Frage auf, unter welchen Bedingungen geltendes Recht sozial wirksam wird. Jede Rechtsordnung ist für ihre Effektivität auf weitgehende Normkonformität der Rechtsunterworfenen angewiesen. Nur insoweit als die Legitimität des Rechts unbestritten und bestimmte Normen als individuell und sozial verbindlich angesehen werden, ist eine Akzep$\tan z$ des geltenden Rechts zu erwarten.

Bereits diese kurzen Hinweise dürften deutlich machen, daß die Berufung auf die Existenz eines Rechtsgefühls von zentraler demokratietheoretischer und rechtspolitischer Bedeutung ist. Der vorliegende Beitrag stellt demgegenüber die kritische 
Frage, inwieweit heute die Bedingungen noch gegeben sind, unter denen sich ein gemeinsames Rechtsgefühl zu entwickeln vermag.

\section{Die Psychologisierung des Recbtsgefübls - ein Mißverständnis}

Wie die Literaturlage zeigt, wird das Wort ,Rechtsgefühl' zur Bezeichnung sehr unterschiedlicher Sachverhalte verwendet. Weitgehende Einigkeit scheint nur hinsichtlich des Sachverhalts zu herrschen, daß als empirischer ,Ort' des Rechtsgefühls das menschliche Individuum gilt.

Diese scheinbare Selbstverständlichkeit ist zunächst von rechtstheoretischem Interesse: Das menschliche Individuum wird seit der Aufklärung als genuiner Träger unveräußerbarer Rechte konzipiert, und die Legitimitätsbasis der Rechtsordnung auf das Zusammenwirken der Individuen im demokratischen Staat zurückgeführt. Aus eben diesem Grunde wird das, was die Individuen als Recht und Unrecht einschätzen, aber auch, was sie von der jeweils bestehenden Rechtsordnung im ganzen und im einzelen halten, wenn nicht unmittelbar rechtlich, so doch rechtspolitisch von Belang.

Aus der topologischen Verankerung des Rechtsgefühls im Indiviuum - sei es als genetische Disposition, als Gewissen, als Intuition, als kognitives Muster, als sozialpsychologisches Einstellungsbündel oder als aufgeklärte Urteilskraft - scheint jedoch für zahlreiche Autoren ein zweites zu folgen, nämlich die primäre Zuständigkeit der Psychologie im Kontext einer humanwissenschaftlichen Beschäftigung mit dem Rechtsgefühl. Das Rechtsgefühl wird als ein primär psychischer Tatbestand gefaßt, den man mit Kategorien der Adlerschen Individualpsychologie (M. Behbinder), der Kognitionspsychologie (Lampe), einer motivorientierten Entscheidungstheorie (Schwinger), oder eines theoretisch eher diffusen psychologischen Vorverständnisses aufzuklären sucht.

Diese psychologische Auffassung unseres Gegenstandes wird jedoch der genannten rechtstheoretischen Problemstellung m. E. nicht gerecht. Die juristische Tradition seit Ibering und Rümelin loziert das Problem des Rechtsgefühls zu Recht im Verhältnis von Individuum und Rechtsordnung, und nicht im Individuum selbst. ${ }^{1}$ Dieses Verhältnis ist ein doppeltes: Insofern als jedes Individuum bereits innerhalb einer Rechtsordnung aufwächst, ist sein Rechtsgefühl notwendigerweise (wenngleich vielleicht gebrochen) auf die bestehende Rechtsordnung bezogen. Und ebenso ist die jeweils bestehende Rechtsordnung hinsichtlich ihrer Legitimität und Effektivität auf das Rechtsgefühl der Rechtsunterworfenen bezogen. Die empirische Analyse dieser beiden Relationen muß sich nun allerdings recht unterschiedlicher Theoriebereiche bedienen: Das erstgenannte Verhältnis erscheint aus soziologischer Perspektive als ein Problem der Sozialisation, das zweitgenannte als ein solches der Partizipation und des rechtlichen bzw. politischen Verhaltens. Beide Problembereiche sind eher sozialwissenschaftlicher denn psy chologischer Art.

In normativer Hinsicht geht es nun gerade um die Beziehung zwischen den beiden Relationen: Wie das Plädoyer von $M$. Kriele eindrücklich zeigt, ist der demokra- 
tische Rechtsstaat um seiner Funktionsfähigkeit willen auf einen ,inneren Zusammenhang von Rechtswissen und Rechtsgefühl, von positivem Recht und Gerechtigkeit" angewiesen. ${ }^{2}$ Die Fortentwicklung der Rechtsordnung als Rechts- (und nicht Willkür-)Ordnung setzt voraus, daß zum mindesten diejenigen, die mit der Anwendung und Fortentwicklung des Rechts befaßt sind, in ihrem Rechtsgefühl mit den tragenden Grundsätzen der Rechtsordnung übereinstimmen. ,Tragende‘ Grundsätze jedoch sind solche, die von der Gemeinschaft der Rechtsunterworfenen als spezifische Qualitäten des Rechts wahrgenommen und geschätzt werden. ${ }^{3}$ M.a.W. nur solange als zwischen den Inhabern der politischen und der judikativen Macht und der großen Mehrheit der Bürger und Rechtsunterworfenen ein gewisser Grundkonsens über Wert und lnhalt der Rechtsordnung besteht - und d. h. in unserem $\mathrm{Zu}$ sammenhang: insoweit ihr ,Rechtsgefühl' genügende Ähnlichkeiten aufweist - sind die Funktionsvoraussetzungen eines demokratischen Rechtsstaates intakt. Es scheint mir daher sinnvoll, den schillernden Begriff des Rechtsgefühls zur Bezeichnung genau dieses Problems des (doppelten!) Verhältnisses von Bürgern bzw. Rechtsunterworfenen und Rechtsordnung zu verwenden. Legitimitätsglaube wie auch Normkonformität und ,Rechtswille' (i.S. Krieles) sind - zum mindesten innerhalb heute noch recht unbestimmter Grenzen - von kognitiven und affektiven Voraussetzungen auf seiten der beteiligten Individuen abhängig, über deren Inhalt und Genese bisher nur wenig bekannt ist.

Im Rahmen dieses Beitrags steht die sozialisationstbeoretische Betrachtung des Rechtsgefühls im Vordergrund. Wie auch immer man das Rechtsgefühl ${ }^{4}$ (oder breiter: die handlungsrelevanten psychischen Gegebenheiten im Verhältnis zur Rechtsordnung überhaupt) individualpsychologisch deuten mag, wir können annehmen, daß die für die Rechtsgemeinschaft relevanten individuellen Dispositionen nicht angeboren, sondern aufgrund vorhandener Anlagen durch die Verarbeitung von Informationen und sozialer Erfahrungen erworben bzw. entwickelt worden sind. ${ }^{s}$

So plausibel diese allgemein gehaltene Aussage auch sein mag, die empirische Evidenz von Effekten politischer oder rechtlicher Sozialisation ist alles andere als zufriedenstellend. Dies liegt nicht nur am noch relativ sporadischen Charakter der empirischen Forschungsergebnisse. Auch dort wo - wie z. B. in der Wahlforschung - ein überaus breites empirisches Datenmaterial vorliegt, sind die Befunde mebrdeutig, d.h. mit sehr unterschiedlichen Erklärungsansätzen kompatibel. ${ }^{6}$ Für die uns hier interessierenden Bereiche von Rechtsbewußtsein, Rech tsgefühl und Rechtswillen liegen $\mathrm{m}$. W. noch kaum systematische empirische Untersuchungen vor, was schon deshalb nicht verwundern kann, weil die begrifflichen Konstrukte noch sehr vielfältig und unbestimmt sind. Es scheint sich bisher eher um Postulate von Juristen als um nachweisbare Sachverhalte zu handeln. Das trifft insbesondere für das Konstrukt ,Rech tsgefühl' zu, welches im wesentlichen als durch Introspektion von Juristen gewonnen erscheint und möglicherweise vor allem ein Ergebnis professioneller Sozialisationsprozesse darstellt. Zum mindesten unter normativen Gesichtspunkten (vgl. Kriele) ist ein enger Zusammenhang zwischen Rechtsbewußtsein, Rechtsgefühl und Rechtswillen zu postulieren, der auch sozialisations- und 
handlungstheoretisch plausibel ist: Insoweit als kognitive und affektive Erfahrungen zum Aufbau einer gleichzeitig kognitiven und motivationalen Struktur mit Bezug auf das geltende Recht, die es stützenden Einrichtungen und seine werthaften Grundlagen führt, ist durchaus ein Zusammenhang zwischen Wissen, Wertschätzung und Willen zu ihrer Aufrechterhaltung zu vermuten. Es ist jedocb eine offene Frage, inwieweit und unter welchen Bedingungen derartige, in der Theorie des demokratiscben Recbtsstaats prinzipiell vorausgesetzte Dispositionen tatsäcblicb sicb entwickeln können.

Unter den in Frage stehenden Begriffen erscheint derjenige des Recbtsbewußtseins der empirischen Sozialforschung grundsätzlich am leichtesten zugänglich, doch handelt es sich auch hier um ein noch recht diffuses Konstrukt. In eigenen Untersuchungen ${ }^{7}$ haben wir unterschieden zwischen (1) dem tatsächlichen (normkonformen) Wissen der Steuerzahler in steuerlichen Belangen, (2) der Einschätzung des eigenen Wissens, (3) der Einschätzung des Wissens und der Handlungsmöglichkeit der Finanzbeamten, (4) der Einschätzung der Behörde Finanzamt im Vergleich zu anderen Behörden. Gegenstand dieser Untersuchung war lediglich ein Ausschnitt der für die Bürger relevanten Teile der Rechtsordnung. Ob das ,Rechtsbewußtsein“ als verallgemeinerte kognitive Struktur oder eher als bereichsspezifische Orientierungen auftritt, ist noch völlig offen.

Im vorliegenden Zusammenhang dürfte der empirische Befund von Interesse sein, daß für die Bereitschaft der Steuerzahler, ihre eigenen Interessen wahrzunehmen, die Einscbätzung des eigenen Wissens bedeutender als das tatsächliche Wissen ist. Darüber hinaus haben wir zahlreiche Hinweise, daß die Einstellung zu Behörden im allgemeinen mit einer Einschätzung der eigenen Handlungsfähigkeit als genereller Disposition zusammenhängt. ${ }^{8}$ In der Terminologie von Scbwinger: Je höher die Selbsteinschätzung der Fähigkeit zur Umweltkontrolle, desto positiver ist das Verhältnis zu Behörden und anderen Großorganisationen, denen man sich beim Fehlen entsprechender Dispositionen tendenziell hilflos ausgeliefert erfährt.

In einer früheren Untersuchung zur sozialpsychologischen Bedeutung sozialer Sicherungssysteme hat der Verfasser die zunächst überraschende Erfahrung gemacht, daß Antworten auf spezifische sozialpolitische Fragen (z.B. die Stellungnahme zu dem das dynamische Rentenprinzip ansprechenden Item „Die Renten steigen mit der Zeit immer genau so wie die Löhne und Gehälter") weniger vom tatsächlichen Informationsstand, als von generalisierten Einstellungen, hier einer Skala "Vertrauen zum Staat", abhängig sind. Eine solche Vertrauensdisposition erschien jedoch damals (1963) vergleichsweise wenig ausgeprägt vorhanden, Mißtrauen und ein damit hoch korrelierendes Anspruchsdenken erwiesen sich als verbreiteter. $^{9}$

Diese und ähnliche punktuelle Befunde anderer Autoren haben offensichtlich etwas mit dem hier in Frage stehenden Problemkomplex zu tun, aber sie lassen keine eindeutige, stabile Struktur erkennen. Wahrscheinlicher erscheint, daß Einstellungen zu rechtlichen und politischen Gegebenheiten lediglich bei Personen, die einen regelmäßigen, meist beruflich vermittelten Kontakt zu diesen Gegebenheiten haben (Juristen, Angehörige des öffentlichen Dienstes, Politiker) zu mehr oder 
weniger eigenständigen kognitiven Strukturen werden, während sie beim Rest der Bevölkerung einerseits auf sehr allgemeiner Ebene von anderen kognitiven Strukturen abhängig erscheinen oder schlicht auf einem stark flukturierenden Meinungsniveau verbleiben. Es erscbeint demzufolge als wabrscbeinlich, daß beute Recbtsbewußtsein und Recbtsgefübl außerbalb der juristiscben Profession relativ unbestimmt bleiben. ${ }^{10}$ Daß hierunter auch der Rechtswille leidet, wie Kriele vermutet, ist nicht unplausibel.

Die wenig befriedigenden Befunde auf der Erfahrungsebene der Individuen geben Anlaß, der Frage der Vermittlung zwischen Rechtsordnung und Indiviuum Beachtung zu schenken. Ich konzentriere mich dabei auf die erste Relation im einleitend skizzierten Verhältnis, also auf die Frage, wie die Individuen Bestand, Wertigkeit und Wirksamkeit der Rechtsordnung erfabren können, was offensichtlich eine Bedingung für die Entstehung von Rechtsbewußtsein, Rechtswillen und Rechtsgefühl ist. Die zweite Relation, welche die Nutzungs- und Beeinflussungsmöglichkeiten der Rechtsordnung durch die Individuen in ihrer Rolle als Stimmbürger, Rechtsuchender, Steuerzahlen, Anspruchsberechtigter etc. betrifft, kann nur insoweit einbezogen werden, als sie für die erste Relation von Belang ist. In diesem Zusammenhang sei auf zwei historische Trends hingewiesen, die für die Erklärung der gegenwärtig zu vermutenden Unbestimmtheit von Rechtsbewußtsein und Rechtsgefühl aus soziologischer Sicht bedeutungsvoll erscheinen, nämlich die zunehmende Verrechtlichung aller Lebensbereiche und das zunehmende Bewußtsein von der Kontingenz des Rechts.

\section{Verrechtlicbung und Recbtsgefübl}

Aus der Sicht des Juristen gibt es kaum irgendwelche soziale Verhältnisse ohne rechtliche Qualität. Sie mag sich ausdrücklich aus Gesetzen ergeben, sie kann aber auch erst durch einen Rechtsstreit zum Problem werden, wobei dann die sozialen Tatbestände durch Subsumption oder Analogiebildung ,verrechtlicht werden. In der Perspektive der juristischen Laien dagegen, in unserem Alltagsverständnis, tritt der rechtliche Charakter der Sozialverhältnisse, in die wir uns begeben, nicht in den Vordergrund, er bleibt latent. Unsere alltägliche Zuwendung zur Welt ist durch Gewohnheiten, durch Vertrauen und durch die Erwartung der Stabilität gegebener Verhältnisse gekennzeichnet. " Innerhalb gegebener Kontexte ist soziales Handeln weitgehend unproblematisch. Hier wird ein situationsbezogenes Wissen wirksam, das nur in den charakteristischen Ausnahmefällen einer thematischen Kommunikation über Recht (z. B. bei einem schriftlichen Vertrag oder bei juristischer Tätigkeit) explizit rechtsbezogen ist. Üblicherweise wird Recht thematisch erst, wenn der gewohnte Lauf der Dinge unterbrochen ist, wenn Erwartungen enttäuscbt werden. Auch dann ist die Orientierung am Recht noch keineswegs selbstverständlich.

1. Der ganz überwiegende Teil unserer Erwartungsenttäuschungen im Verhältnis zu anderen Rechtssubjekten wird in der Form eines individuellen Konflikts erlebt, verarbeitet oder auch verdrängt. 
2. Der frustrierte Partner eines (immer noch latenten) Rechtsverhältnisses kann seiner Enttäuschung Ausdruck geben, und dann wird es i.d.R. zu einer Art Neuverhandlung zwischen den Partnern kommen. Hier entsteht ein sozialer Konflikt, der aber nicht in der Form des Rechts ausgetragen wird, sondern als Versuch, das gestörte Interaktions- und Vertrauensverhältnis wieder herzustellen. Dabei ist die Berufung auf Gerechtigkeitsargumente keineswegs selten. Gelegentlich spielen auch rechtliche Argumente eine Rolle, doch bleiben sie so lange der sozialen Interaktion untergeordnet, als kein Rechtsstreit angedroht wird.

3. Sobald rechtliche Konsequenzen angekündigt werden (und zwar unabhängig von deren Berechtigung) tendiert die soziale Interaktion zu einer Veränderung. Der Konflikt erhält nunmehr eine spezifisch recbtlicbe Färbung, er tritt aus dem Bereich des Privaten in denjenigen des Offentlichen. In der Regel werden nun Dritte - beispielsweise die Polizei oder ein Anwalt - eingeschaltet, die von Berufs wegen gehalten sind, den Konflikt unter rechtlichen Gesichtspunkten zu betrachten. Diese ,Verrechtlichung' ist zumeist Folge, gelegentlich aber auch Ursache eines Zusammenbruchs der alltäglichen Kommunikation, die durch einen gewissen Vertrauensvorschuß und die Anerkennung von Selbstverständlichkeiten zu kennzeichnen ist. Nun gilt nur noch das, was man beweisen kann, und was Gesetz und Rechtsanwender dazu sagen. An die Stelle einer Kooperationsorientierung tritt eine strategische oder zum mindesten taktische Einstellung zum Partner als potentiellem Gegner. Das Vertrauen wird durch ein kalkuliertes Mißtrauen ersetzt. ${ }^{12}$

Unter Verrecbtlicbung sei also der Umstand verstanden, daß soziale Sachverhalte unter dem Gesichtspunkt des Rechts betrachtet und verhandelt werden. Wie skizziert, bleibt der Rech tscharakter privatrechtlicher Sozialverhältnisse i.d.R. latent. ${ }^{13}$ Das ist nicht in gleichem Umfang für öffentlich-rechtliche Verhältnisse zu vermuten.

Hier ist zum mindesten einer der Interaktionspartner aus beruflichen Gründen, an Recht und Gesetz gebunden' und neigt von seiner beruflichen Sozialisation her dazu, die Sachverhalte unmittelbar unter Rechtsgesichtspunkten zu betrachten. Insoweit dies auf beiden Seiten der Fall ist, also ty pischerweise bei einer Kommunikation unter Juristen, ist zwar keine Einigkeit, aber doch die Anerkennung gewisser juristischer Selbstverständlichkeiten zu erwarten, die den Laien häufig gerade nicht bekannt sind. Hieraus resultiert u. a. die charakteristische Asymmetrie des Verhältnisses zwischen Verwaltungen und ihrem Publikum ${ }^{14}$, die vor allem im Bereich sozialstaatlicher Leistungen zur Kritik an ihrer Verrechtlichung geführt hat. ${ }^{15}$ Der häufig pauschal erhobene Vorwurf einer ,Verrechtlichung der Sozialpolitik' ist allerdings nur mit Bezug auf bestimmte soziale Leistungen qualifiziert: Uberall dort, wo die soziale, d.h. auf personenbezogenem Vertrauen beruhende Interaktion Voraussetzung der Wirksamkeit bestimmter Sozialleistungen ist, kann deren Verrechtichung zu nachteiligen Folgen führen. Demzufolge ist z. Zt. die Verrechtlichung der Medizin in besonderem Maße umstritten. ${ }^{16}$ In anderen Bereichen, etwa dem des Arbeitsschutzes oder der Sozialversicherung, ist deren Verrechtlichung zweifellos Bestandteil des sozialpolitischen Fortschritts.

Betrachten wir diese Befunde in der Perspektive unserer Frage, wie das Recht heute dem juristischen Laien vermittelt und von ihm erfahren wird, so erscheint 
eine gewisse Ambivalenz der unmittelbaren Erfabrung mit dem Recht vorprogrammiert. Die wohltätigen, die ordnungsstiftenden und die jene unproblematischen Alltagserfahrungen ja erst ermöglichenden Funktionen des Rechts bleiben latent; manifest erscheint das Recht in der Gestalt des die soziale Alltagserfahrung Verfremdenden, als in ihrer Struktur unbekannte und damit unheimliche Ordnung, als schicksalsmächtiger Richterspruch oder als undurchschaubare Bürokratie. Insofern wir unter Rechtsgefühl eine präreflexive oder selbst gewissenmäßig gefilterte Empfindung für das Richtige oder Billige verstehen, besteht unter den vorherrschenden Bedingungen der Erfahrbarkeit des (positiven) Rechts nur eine vergleichsweise geringe Chance zu bestätigenden unmittelbaren Erfahrungen. Selbst wenn bei vollständiger Kenntnis der Zusammenhänge eine Konkordanz von Rechtsempfinden und Verfahrensergebnis zu erwarten wäre, bewirkt doch allein schon die Intransparenz des Verfahrens selbst Mißbilligung und nicht selten Mißtrauen.

Man wird zu Recht einwenden, daß dies nicht der einzige Weg sei, auf dem juristischen Laien die Rechtsordnung vermittelt wird. Eine größere Vertrautheit mit rechtlichen und administrativen Gegebenheiten ergibt sich für wachsende Bevölkerungsteile aus einer Sekundärwirkung der zunehmenden Verrechtlichung, nämlich ihrem beruflichen Umgang mit Rechtssachverhalten. Angehörige des öffentlichen Dienstes etwa zeigen deutlich geringere Mißtrauens- und höhere Vertrauenswerte mit Bezug auf Staat und Verwaltung, wobei dies mit einer höheren Bewußtheit für die Fehleranfälligkeit, aber auch Beeinflußbarkeit administrativer Prozesse einhergeht. Weniger überzeugend sind die bisherigen Ergebnisse politischer Bildung. Dies mag teilweise an der Art und Weise ihrer Institu tionalisierung liegen, hat aber zweifellos auch tieferliegende Gründe: Politiscbe Sozialisation, d. h. die Vermittlung von politisch relevanten Kognitionen und Motiven erfolgt überwiegend beiläufig, etwa in der familieninternen Kommunikation über massenmedial vermittelte politische Ereignisse, durch Diskussionen unter Altersgenossen und Berufskollegen, nicht zuletzt aber auch - und zwar vor allem in der Dimension ethischen Handelns - durch Vorbildlernen, d. h. durch Identifikation mit und Imitation von geschätzten Personen. ${ }^{17}$

Diese Einflüsse sind ihrem Ursprung wie auch ihrer Richtung nach i.d.R. beterogen (wenngleich in schichtspezifisch variablem Maße), so daß es nicht überraschen kann, wenn der gegenwärtige Forschungsstand zur politischen Sozialisation von ,widersprüchlichen subjektiven Aneignungs- und Ausdrucksformen von politischgesellschaftlicher Realität "18 ausgeht. Diese Widersprüchlichkeit führt vermutlich zu der beschriebenen Unbestimmtheit des Recbtsbewußtseins, da die heterogenen Sozialisationseinflüsse keine prägnante Ausformung politikbezogener motivationaler und kognitiver Strukturen ermöglichen.

Es liegt nahe, unserer Analyse eine soziologistische Verkürzung der Problemstellung vorzuwerfen. Kann und muß nicht doch eine innere und durch die Heterogenität sozialer Erfahrungen nicht zerstörbare Disposition des Menschen auf Ordnung (Rümelins ,Ordnungstrieb'), auf soziale Harmonie, oder kognitive Konsistenz auf Gemeinschaft oder Gerechtigkeit angenommen werden? Greift nicht Iherings Kritik an den „Nativitätstheorien“" insofern zu kurz, ais ja auch der Aufbau eines Rechtsbewußtseins oder Rechtsgefühls auf bestimmten entweder angeborenen oder doch im Zuge der Ontogenese reifenden Dispositionen beruhen muß? 
Es sei in diesem Zusammenhang auf die anthropologischen Studien von D. Claessens hingewiesen, die unser Thema unmittelbar betreffen: Die Zurückdrängung angeborener Mechanismen im Prozeß der Hominisation hinterläßt „formale Prinzipien von Instinkten oder Instinkt-Systemen, die inhaltlich entleert im Menschen von heute weiter wirksam sind“. ${ }^{19}$ Sie erscheinen als die anthropologische Grundlage jener Dispositionen zum Aufbau kognitivemotionaler Ordnungen, einer ,Sinntendenz', einer sozusagen konstitutionellen Unfähigkeit, die Welt als Chaos zu erfahren. Hierauf führt Claessens die Bereitschaft, ,Geltung anzuerkennen, zurück, wie sie sich in allen menschlichen Kulturen manifestiert. Dennoch wäre es ein Trugschluß, wollte man aus diesen anthropologischen Einsichten auf die Gewährleistung eines unverlierbaren Rechtsgefühls schließen. In seinem jüngsten Werk begründet Claessens vielmehr die These, ,daß sich in der Freisetzung des Menschen durch sich selbst ein evolutionäres Handikap offenbart, das den Kontrapunkt zu seinen Distanzierungstechniken darstellt ..., daß neben der Hauptaabigkeit zur Distanzierung von der ,alten Natur' das Hauptdefizit des Menschen seine evolutionär bedingte Unfähigkeit ist, zum Organisieren großer Populationen und den sich dabei unvermeidlich ergebenden Komplikationen ein direktes emotionales, d.h. unmittelbar motivierendes Verhältnis $\mathrm{zu}$ finden". ${ }^{20}$

Genau der Sachverhalt des „Organisierens großer Populationen“ ist es jedoch, mit dem es der moderne Rechts- und Sozialstaat zu tun hat. Er führt, dies ist die These des dritten Teils, zu Verhältnissen, in denen ,die Grundthese der Rechtsanthropologie ...: daß das Recht strukturell an den Einzelmenschen gebunden ist und gebunden sein muß, sofern es Anspruch erhebt, richtiges Recht zu sein ${ }^{\text {،21 }}$ ihrer empiriscben Bedingungen tendenziell beraubt wird, so daß das normative Postulat mehr und mehr in der Luft hängt.

\section{Wandel des Recbts und Rechtsgefübl}

Die ambivalenten Wirkungen der Verrechtlichung auf die Vermittlung zwischen Individuum und Rechtsordnung werden erst voll verständlich, wenn wir einen dritten Problemkomplex in die Betrachtung einschließen: Die Expansion der Recbtsstaatlicbkeit und ihre Folgeprobleme.

Der moderne Rech tsstaat kontinentaleuropäischer Prägung beruht auf gesatztem Recht und dessen verfahrensmäßig geregelter Änderungsform. Im Vergleich zur Rechtstradition des gemeinen Rechts ergibt sich hier eine höhere Änderbarkeit des Rechts und seine geringere Bindung an Sitte und Gewohnheit. Dies trifft in besonderem Umfange zu, wenn sich der Gedanke der Rechtsstaatlichkeit mit demjenigen der repräsentativen (d.h. indirekten) Demokratie und der Institution eines überwiegend juristisch geschulten Berufsbeamtentums verbindet, wie dies für die Bundesrepublik Deutschland charakteristisch ist. Hier sind alle Voraussetzungen für eine zunehmend instrumentelle und konstruktive Handbabung des Recbtssetzungs- (und d.h. heute überwiegend: Rechtsänderungs) verfabrens gegeben, das sich von Sitte und Gewohnheit abkoppelt und häufig nicht einmal den von Berufs wegen mit be- 
stimmten Rechtsmaterien Befaßten, geschweige denn der Öffentlichkeit Zeit läßt, ein mit den positiven Rechtsregelungen übereinstimmendes Rechtsbewußtsein zu entwickeln. F. C. v. Savigny hatte vor dieser Tendenz der Rechtsentwicklung bereits im Zusammenhang mit der Kodifikation des bürgerlichen Rechts gewarnt. ${ }^{22}$

Die deutsche Rechtswissenschaft des 19. und 20. Jahrhunderts hat - nicht zuletzt unter dem Einfluß lherings - die generalisierende, vereinheitlichende und damit der Nivellierung sozialer Verhältnisse dienende Funktion des modernen Rechts bejaht, und die Erfahrungen der Rechtsperversion im Dritten Reich haben in der Bundesrepublik zu einem besonderen Bemühen um Rechtsstaatlichkeit geführt. Ausdruck dieses Bemühens ist eine wachsende Zahl von Gesetzen und Rechtsverordnungen: Nach einer Feststellung der Bundesregierung waren am 18. Februar 19771480 Gesetze und 2280 Rech tsverordnungen allein auf Bundesebene in Kraft, dazu kommt das Landesrecht ${ }^{23}$ und zunehmend auch das innerstaatlich verbindliche Recht der Europäischen Gemeinschaft. ${ }^{24}$ Noch stärker als der Gesetzesbestand wächst die Zahl der Gesetzesänderungen, wie allein schon die Umfangzunahme des Bundesgesetzblattes und ähnlicher Publikationsorgane zeigt. Diese „Verrechtlichungstenden $z$ " als immer umfassendere und detailliertere Regelung des öffentlichen Lebens führt zu einer ,,Selbstgefährdung des Rechts “25, insofern als jeglicher Uberblick und auch die Entwicklung eines angemessenen Rechtsbewußtseins und Rechtsgefühls als „,sedimentierte Rechtserfahrung ${ }^{\text {"26 }}$ selbst bei den juristisch Gebildeten immer mehr erschwert wird. Die Intensivierung des Rechtsstaats scheint einen abnehmenden, wenn nicht gar negativen Grenznutzen zu produzieren. Es ist, als ob die Qualität des Rechts mit der quantitativen Zunahme rechtlicher Normierung sich verändere.

$R$. Voigt unterscheidet in diesem Zusammenhang zwischen Vergesetzlichung, Bürokratisierung und Justizialisierung, um den gleichzeitigen Einfluß der drei klassischen Staatsgewalten auf den Verrechtlichungsprozeß zu verdeutlichen. ${ }^{27}$ Aber auch die Rechtsgenossen selbst tragen zur Verrechtlichung bei, indem sie ihre Verhältnisse zunehmend unter Rechtsgesichtspunkten wahrnehmen und strukturieren. Dabei sollte nicht unbeach tet bleiben, daß der ganz überwiegende Teil der, Gesetzesflut ${ }^{‘}$ nicht das bürgerliche Recht, sondern öffentliche und quasi-öffentliche Verhältnisse betrifft. ${ }^{28}$ Der schweizer Staatsrechtler Hans Huber bemerkte hierzu bereits 1953:

„Die neuen Rechtsstrukturen und Rechtsgebiete entfernen sich vom ethischen Gravitationszentrum des Rechts. Das zeigt sich bis hinein in die Begriffsbildung, etwa des Wirtschaftsrechts. Die verwendeten Begriffe sind mehrheitlich traditionslose Zweckschöpfungen, die Rechtssätze und Institutionen sind gelöst von jeder Vorformung durch andere gesellschaftliche Ordnungen wie Sitte und Konvention, Moral und Religion.... Gewiß werden die abstrakten Ziele, namentlich die sozialpolitischen, weniger die wirtschaftspolitischen, manchmal mit der Zeit vom Kollektivbewußtsein akzeptiert. Damit jedoch das Recht der Massengesellschaft naherücken würde, wäre erforderlich, daß nicht nur die Ziele, sondern auch seine Bestandteile, z. B. seine Solidaritätspflichten bis zu den verschiedenen Steuerpflichten für sozialen Ausgleich, in jene Schichten des Bewußtseins eingehen würden. Es ist jedoch zu befürchten, daß das Recht der Massengesellschaft auch in der Zukunft, abgesehen von seinen abstrakten Zielen, den Widerhall in den Herzen nicht finden werde, den das Recht zu seiner wahren Geltung bedarf und den ein 
Recht leichter findet, das den anderen gesellschaftlichen Ordnungen nahesteht, z. B. ,moraldurchtränkt' ist. Namentlich wo das den neuen Rechtsstrukturen entsprechende Verwaltungshandeln ein solches der Bureaukratie ist, haben jene die größte Mühe, anders denn als Zwang hingenommen zu werden. " 29

Diese $m$. E. richtigen Einschätzungen erlauben uns, die Problematik der Verrechtlichung präziser zu fassen. Es ist nicht so sehr der Umstand, daß bestimmte Sachverhalte überhaupt unter Rech tsgesichtspunkten erörtert werden, der das Unbehagen auslöst, sondern es ist eine Art Qualitätsänderung des Recbts selbst, die zu einer immer schärferen Trennung von Recht und Moral oder Anspruch und Vertrauen führt. Das Recht entweicht sozusagen aus der Sphäre des zwischenmenschlichen Verkehrs in eine gesonderte Schicht der Wirklichkeit. Das Recht verliert seinen Charakter als Element des praktischen All tagsbewußtseins und wird zu einer immer exklusiveren Angelegenbeit von Spezialisten. Der Konsens über Recht und Unrecht wird immer unbestimmter, und zwar von zwei Seiten her: zum einen infolge der Rechtsentwicklung selbst, die sich um das vorhandene Rechtsbewußtsein immer weniger kümmert, Gesetzgebung also als eine konstruktive oder bestenfalls politische Angelegenheit ansieht, die im Rahmen der repräsentativen Demokratie auch ohne Volksbeteiligung vonstatten gehen kann: Legitimation durcb Verfabren. ${ }^{30}$ Die Fülle und insbesondere die häufigen Änderungen von Gesetzen lassen eine einigermaßen sichere Aneignung der wichtigsten Rechtsprinzipien im Verständnis auch der interessierten Laien nicht mehr zu, ja selbst Juristen geben immer häufiger zu erkennen, daß ihnen nur noch die Fundstelle, aber nicht mehr der Inhalt der einschlägigen Rechtsnormen bekannt ist.

Zum anderen tendiert aber auch das individuelle Rechts-, oder vielleicht besser Billigkeits- bzw. Gerechtigkeitsbewußtsein dazu, sich stärker zu diversifizieren, wozu Gruppenmeinungen in nicht unerheblichem Maße beitragen dürften. Dieser Prozeß ist durch den ersten unmittelbar mitbedingt. Wo das Wissen, was rechtens ist, unmöglich wird, treten Meinungen an dessen Stelle. Und Meinungsbildungsprozesse sind in hohem Umfange von Sozialbeziehungen abhängig, die man zumeist nur mit seinesgleichen pflegt. Daß einige Formen der Steuerhinterziehung, der Vorteilserschleichung, der Kundentäuschung oder auch Abtreibung bzw. Ehebruch in bestimmten Kreisen bestenfalls als Kavaliersdelikt angesehen werden, hat mit derartigen sozialen Verstärkungsprozessen zu tun, die sich andererseits - etwa unter Theologen oder Alternativen - auch zu besonders ausgeprägten Moralisierungen steigern können. Das Problem ist ein Unbestimmtwerden des gemeinsamen moralischen Bewußtseins, das zunehmend seinen rechtlichen Kern zu verlieren scheint. Es ist schwierig, das Neue gegenwärtiger Entwicklungstendenzen genauer $2 \mathrm{u}$ beschreiben. Natürlich hat es schon immer Subkulturen mit eigenen Normen gegeben, die sich gegen das vorherrschende Rechtsbewußtsein als mehr oder weniger resistent erwiesen haben. Das bereits von Hegel gesehene tendenzielle Auseinandertreten von Legalität und Moralität ist ebenfalls eher als eine Vorstufe zu betrachten, denn wie bereits angedeutet, geht es weniger um den Konflikt zwischen einer abweichenden Rechtsüberzeugung und der herrschenden Rechtsordnung, als um ein Unbestimmt- 
werden des Rechtsbewußtseins, den Verlust an Rechtsüberzeugungen schlechthin. Nicht die gelegentlich öffentlichkeitswirksame Berufung auf eine metajuristische Moral, sondern eher ein um sich greifender Opportunismus auch und gerade unter den höheren Bildungsschichten signalisiert das Problem. Vielleicht könnte man es als eine Vergleichgültigkeit des Öffentlicben bezeichnen: Der zunehmend massenmedial vermittelte Modus, in dem Politik und Recht wahrgenommen werden, führt für den Außenstehenden zu einer Überforderung seines Urteilsvermögens. Der Pluralismus der öffentlichen Meinung führt in seiner Vielschichtigkeit und unentscheidbaren Widersprüchlichkeit leicht dazu, unterschiedliche Positionen als gleich gültig, und damit zunehmend als gleichgültig anzusehen. Inwieweit also das Recht noch als Ausfluß einer "lebendigen Sittlichkeit" erfahren und vermittelt werden $\operatorname{kann}^{31}$, bildet den ersten Aspekt der unter dem Titel ,Rechtsgefühl' angesprochenen Problematik.

Der zweite Aspekt bezieht sich auf den Zusammenhang von Rechtsgefühl und Rechtssicberbeit, die sozusagen eine zweite Verteidigungslinie rechtsstaatlicher Legitimation darstellt. Wie an anderer Stelle zu zeigen versucht wurde ${ }^{\mathbf{3 2}}$, bezieht sich das Postulat der Rechtssicherheit auf das Problem der Wirksamkeit der Rechtsordnung. Diese ist nun keineswegs nur von der Effektivität der Normdurchsetzung ${ }^{33}$, sondern ebenso vom Glauben an den ordnenden Charakter des Rechrs abhängig. Eine Ordnung ist nur insoweit verläßlich, als die verbreitete Erwartung in die Wirksamkeit der Ordnung deren Wirksamkeit verbürgt. „Das Vertrauen, das der Einzelne in die vorhandene Regelung des Gemeinschaftslebens will setzen können “34 ist ein weiterer Aspekt des Rechtsgefühls, dessen Bestand in dem Maße gefährdet erscheint, als die Kontingenz von Rechtssetzung, Rechtsanwendung und Rechtsdurchsetzung ins allgemeine Bewußtsein tritt. Die neuzeitliche Gesellschaftsentwicklung ist durch eine ungeheuere Expansion des Möglicben gekennzeichnet, dessen, was die mittelalterliche Philosophie als Kontingentes, d.h. weder Notwen+ diges noch Unmögliches, bezeichnet hat. Was möglich ist, kann auch anders sein, d.h. sein Bestand ist nicht über jeden Zweifel erhaben. ${ }^{35}$ Vor allem aus der Perspektive des juristischen Laien erscheint das Recht immer weniger als Ordnung, mit der man rechnen kann, weil das sichere Rechtsbewußtsein infolge einer Inflation der Regelungen sich auflöst. Aber auch innerhalb der Jurisprudenz schreitet die Spezialisierung in einer Weise fort, die zu ähnlichen Tendenzen führen kann. Typische Phänomene, die das Kontingenzbewußtsein mit Bezug auf das Recht erhöhen, sind etwa die Reduktion sozialer Leistungen, die konkurrierende Anwendbarkeit unterschiedlicher Rechtsnormen infolge der wachsenden Interventionsdichte oder das Wachstum der Schattenwirtschaft. Gerade dieses letzte Phänomen und weitere Formen einer alternativen OKkonomie sind charakteristische Reaktionen auf einen überkomplexen Gesellschaftszustand, wie er durch das Zusammenspiel von Markt und Staat konstituiert wird. ${ }^{36}$ Der Versuch, neue Formen der Bedarfsdeckung im Nahbereich und häufig auch unterhalb expliziter rechtlicher Regelungen auf der Basis von Selbsthilfegruppen und anderer Formen solidarischer Kooperation zu praktizieren, ist vermutlich praktischer Ausfluß jenes „Unbehagens in der Modernität", das sich auch in einer Krise des Rechtsgefühls niederschlägt. ${ }^{37}$ Vielleicht läßt sich 
das Problem auch auf die Pointe bringen, daß die am Verhältnis von Staat und Privatrecht entwickelten Legitimationstheorien des Rechts ihre Plausibilität in einer Situation einbüßen, in der ein immer größerer Teil des gesetzten Rechts der Selbststeuerung des Staates dient, und die Rechtserfahrung des Bürgers vorwiegend aus Verwaltungskontakten stammt.

\section{Zusammenfassung}

Die Berufung auf das Rechtsgefühl ist von zentraler demokratietheoretischer und rechtspolitischer Bedeutung. Im vorliegenden Beitrag wurde nach den empirischen Bedingungen gefragt, unter denen ein gemeinsames Rechtsgefühl sich entwickein kann. In sozialisations-theoretischer Perspektive geht es dabei um die Frage, wie die Individuen, deren Rechtsgefühl in Frage steht, Bestand, Wertigkeit und Wirksamkeit der Rechtsordnung erfahren können. Die hierfür relevanten Bedingungen haben sich, das ist die zentrale These dieses Beitrags, im Zuge der gesellschaftlichen Entwicklung wiederholt gewandelt. In dem Maße, als nicht mehr die friedenstiftenden Funktionen des Rechts in der Form des Privat- und Strafrechts im Vordergrund der Erfahrung des geltenden Rechts stehen, sondern durch den Ausbau der staatlichen Tätigkeit das Verwaltungsrecht (etwa das Steuer-, Bau- und Sozialrecht) zunehmende Alltagsrelevanz erlangt, wandelt sich die vorherrschende Wahrnehmung des Rechts. In dem Maße, als der zunehmende Regelungsbedarf und die Folgeprobleme bereits bestehender Normierung zu einer fortgesetz ten Revision der Rechtsordnung drängen, verändert sich die vorherrschende Vorstellung von Recht als Friedensordnung in die Richtung auf ein konstruktivistisches Rechtsverständnis - das Recht wird zur Steuerungssprache des öffentlichen Sektors. Gleichzeitig wächst beim Juristen das Bewußtsein der Kontingenz und Wandelbarkeit des Rechts, beim Laien das Bewußtsein seiner Intransparenz. In der Folge treten die Rechtsauffassungen der professionellen Jurisprudenz und das Rechtsbewußtsein der Laien tendenziell auseinander, soweit diese überhaupt noch ein Rechtsbewußtsein zu entwickeln imstande sind. Insoweit als diese Diagnosen zutreffen, muß mit einem abnehmenden Grenznutzen des Rechtsstaats gerechnet werden. Es ist daher erforderlich, die Probleme staatlicher Steuerung durch Recht auch zum Gegenstand expliziter rechtswissenschaftlicher Uberlegungen und rechtspolitischer Maßnahmen zu machen. ${ }^{38}$

\section{Anmerkungen}

1 M. Rümelin bemerkt zu Recht: „Die Annahme eines besonderen, gerade in Beziehung auf das Recht wirksamen seelischen Vermögens, enthält nichts weiter als eine völlig willkürliche und unfruchtbare Konstruktion". (Rechtsgefühl und Rechtsbewußtsein, Tübingen 1925, S. 26). - Im Vergleich zur mir bekannten jüngeren Literatur findet sich die prägnanteste Formulierung von Genese und Funktion des Rechtsgefühls immer noch bei $R$. v. Ihering. Seine vielfach zitierte Sentenz ,Nicht das Rechtsgefühl hat das Recht, sondern das Recht hat das 
Rechtsgefühl erzeugt" (zuerst in der Vorrede zu "Der Zweck im Recht") beschreibt nur die gegen die Vorstellung eines angeborenen Rechtsgefühls gerichtete Seite seiner Argumentation. Ihering betont gleichzeitig, ,daß unser Rechtsgefühl, (scil. auch) wenn es seine Nahrung von außen, von den Rechtssätzen und Einrichtungen, die aufgestellt sind, bezieht, schließlich denselben überlegen wird", so daß ein sich (aufgrund des "Abstraktionsvermögen des menschlichen Geistes") fortentwickelndes Rechtsgefühl das bestehende Recht, in Frage zu stellen, über sich hinauszutreiben vermag. (R.v. Ihering, Öber die Entstehung des Rechtsgefühls. In : ders., Der Kampf ums Recht. Ausgewählte Schriften, hrsg. v. Ch. Rusche, Nürnberg 1965, S. 275-302, Zitate S. 296). Vgl. hierzu auch H. Schelsky, Das Ihering. Modell des sozialen Wandels durch Recht - Ein wissenschaftsgeschichtlicher Beitrag. In: JB f. Rechtssoziologie und Rechtstheorie, Bd. 3: Zur Effektivität des Rechts. Düsseldorf 1972, S. 47-86.

$2 \mathrm{M}$. Kriele, oben S. 29. Vgl. in diesem Zusammenhang auch M. Rehbinder, Rechtskenntnis, Rechtsbewußtsein und Rechtsethos als Probleme der Rechtspolitik. In: JB f. Rechtssoziologie und Rechtstheorie, Bd. 3, a.a.O., S. 25-46.

3 Diese tragenden Grundsätze haben $m$. E. nicht nur mit dem Prinzip der Gerechtigkeit, sondern auch mit demjenigen der Rechtssicherheit zu tun, wie bereits Rümelin (a.a.O., S. 65 ff.) hervorgehoben hat.

4 Obwohl im folgenden bewußt von einer dezidierten Interpretation des Rechtsgefühls abgesehen wird, seien drei Hauptdimensionen - wie sie der Verfasser sieht - hier erwähnt: (1) Rechtsgefühl als intuitive Einschätzung, daß bestimmte Handlungen oder Situationen mit Normen der Rechtsordnung (nicht) übereinstimmen. In dieser Version kommt der Begriff denjenigen des (Un) Rechtsbewußtseins nahe. (2) Rechtsgefühl, daß man sich auf das Recht verlassen kann (Rechtssicherheitsgefühl). (3) Rechtsgefühl als Gefühl, daß (in der Welt, im konkreten Fall) das sich Ereignende im Kontext einer richtigen, gerechten Ordnung geschieht, daß einem, recht geschieht' (Gerechtigkeitsglaube).

5 Den entscheidenden Punkt hebt E. Riezler hervor: „Darin, daß jedes inhaltich irgendwie bestimmte Rechtsgefühl unter wesentlicher Mitwirkung kognitiver Vorstellungen zustande kommt, die ihrerseits historisch und empirisch bedingt sind, erblicke ich den Haupteinwand gegen die Annahme eines ursprünglich angeborenen Rechtssinns." (Das Rechtsgefühl Rechtspsychologische Betrachtungen. 3. A. München 1969, S. 43.).

$6 \mathrm{Vgl}$. zusammenfassend W. Jagodzinski, Sozialstruktur, Wertorientierung und Parteibindung: zur Problematik eines Sozialisationsmodells. In: Zeitschrift für Soziologie 10 (1981) S. 170-191.

7 D. Grunow, F. Hegner, F.-X. Kaufmann, Empirische Sozialforschung in der Verwaltung: Zum Verhältnis von Steuerzahler und Finanzamt. In: Verwaltungsarchiv 66 (1975) \$. 133. - Dies., Steuerzahler und Finanzamt, Frankfurt/New York 1978.

8 Steuerzahler und Finanzamt, S. $71 \mathrm{f}$.

9 Vgl. F.-X. Kaufmann, Sicherheit als soziologisches und sozialpolitisches Problem - Untersuchungen zu einer Wertidee hochdifferenzierter Gesellschaften. Stuttgart 2. A., 1973, S. $305 \mathrm{ff}$.

10 Diese Vermutung wird durch Theoreme der Gesellschaftstheorie gestützt: Bereits E. Durkheim (De la division du travail social, zuerst Paris 1893) machte auf ein fortschreitendes Unbestimmtwerden des Kollektivbewußtseins im Zuge der gesellschaftlichen Arbeitsteilung aufmerksam. Der Sachverhalt selbst wurde insbesondere von A. Gehlen, Die Seele im technischen Zeitalter - Sozialpsychologische Probleme der industriellen Gesellschaft (Reinbek 1957) eindrücklich analysiert. Die gegenwärtigen theoretischen Anstrengungen von N. Luhmann können nicht zuletzt als Versuche verstanden werden, eine Gesellschaft zu denken, der das bürgerliche Subjekt abhanden gekommen ist.

$11 \mathrm{Vgl}$. hierzu insbesondere die Analyse von A. Schütz/Th. Luckmann (Strukturen der Lebenswelt, Neuwied und Darmstadt 1957) und H. Garfinkel (Studies of the Routine Grounds of Everyday Activities, in: ders., Studies in Ethnomethodology, Englewood Cliffs 1967, S. 35-75). - Uber den Umgang mit Vertrauen unter den Bedingungen zunehmender gesellschaftlicher Komplexität vgl. N. Luhmann, Vertrauen - Ein Mechanismus der Reduktion sozialer Komplexität. Stuttgart 1968.

12 Eine genauere Analyse dieses Sachverhalts gibt G. Ellscheid, Die Verrechtlichung sozialer Beziehungen. In: Neue Hefte der Philosophie 17, Recht und Moral. Göttingen 1979, S. 3761. Als Oberblick über die hinsichtlich ihrer Konnotationen recht diffuse, Verrechtlichungs- 
debatte' sei auf die von R. Voigt herausgegebenen Sammelbünde „,Verrechtlichung“" (Königstein/Ts. 1980) und "Abschied vom Recht" (Frankfurt a.M. 1983) hingewiesen.

13 Wo diese Latenz im Normalfall nicht mehr gegeben ist, wie neuerdings im Bereich des Familien- und Eherechts (als Folge der gesetzlichen Reformen oder als Ergebnis eines grundsätzlichen Einstellungswandels zum Recht?) verändern sich auch die Verhaltensweisen i. S. einer stärker kalkulierenden Haltung.

$14 \mathrm{Vgl}$. D. Grunow, Alltagskontakte mit der Verwaltung, Frankfurt/New York 1978, - F. Hegner, Das bürokratische Dilemma - Zu einigen unauflöslichen Widersprüchen in den Beziehungen zwischen Organisation, Personal und Publikum. Frankfurt/New York 1978.

$15 \mathrm{Vgl}$. H. Achinger, Sozialpolitik als Gesellschaftspolitik - Von der Arbeiterfrage zum Wohlfahrtsstaat. 2. A. Frankfurt 1971, S. 84 ff. - F. Tennstedt, Zur Okonomisierung und Verrechtlichung in der Sozialpolitik. In: A. Murswieck $(\mathrm{Hg}$.), Staatliche Politik im Sozialsektor, München 1976, S. 139-165.

16 Vgl. F.-X. Kaufmann (Hg.), Arzdiches Handeln zwischen Paragraphen und Vertrauen, Düsseldorf, 1984.

$17 \mathrm{Vgl}$. G. Stachel/D. Mieth, Ethisch handeln lernen - Zu Konzeption und Inhalt ethischer Erziehung. Zürich/Köln 1978.

18 Ch. Kulke, Politische Sozialisation. In: Handbuch der Sozialisationsforschung, hrag. v. K. Hurrelmann und D. Ulich, Weinheim und Basel. 2. A. 1982, S. 745-776, Zitat S. 766.

19 D. Claessens, Instinkt, Psyche, Geltung. Bestimmungsfaktoren menschlichen Verhaltens. Eine soziologische Anthropologie. Köln und Opläden, 2. überarb. A. 1970, S. 114.

20 D. Claessens, Das Konkrete und das Abstrakte. Sozjologische Skizzen zur Anthropologie, Frankfurt/M. 1980, S. 17 (Hervorhebung i.O.).

21 E.-J. Lampe, Rechtsanthropologie, Bd. I, Berlin 1970, Vorwort.

22 F. C. v. Savigny, Vom Beruf unserer Zeit für Gesetzgebung und Rechtswissenschaft. 2.A. Heidelberg 1828.

23 Nach F. Wagener (Aufgaben- und Gesetzesinflation - Ursachen und Folgen. In: Verwaltungspraxis (Schweiz) 1982, S. 6) sind, ,in Baden-Württemberg 280 Landesgesetze, 1100 Rechtsverordnungen und 80.000 Verwaltungsvorschriften in Kraft".

24 Nach mündlicher Mitteilung von Katharina Focke (MdEP) am 16.1.84 hat die EG in einem Zeitraum von 18 Monaten rd. 2000 „Verordnungen" (der Begriff wurde nicht näher definiert) erlassen.

25 Vgl. H. W. Weiß, Verrechtlichung als Selbstgefährdung des Rechts. In: Die öffentliche Verwaltung 31 (1978) S. 601-608.

26 H. Ryffel, zit, nach M. Rehbinder, a.a.O., (FN 2) S. 31.

27 R. Voigt, Mehr Gerechtigkeit durch mehr Gesetz? In: Aus Politik und Zeitgeschichte, Beilage zur Wochenzeitung ,Das Parlament“", B. 21/1981.

$28 \mathrm{Zu}$,Ursachen und Reduktionsmöglichkeiten der Uberfülle von Rechtsvorschriften“" vgl. H. Jellinek, in: Verwaltung und Fortbildung 6 (1978), S. 62-71.

29 H. Huber, Niedergang des Rechts und Krise des Rechtsstaats. In: Demokratie und Rechtsstaat. Festgabe zum 60. Geburtstag von Zaccaria Giacometti, Zürich 1953, S. 59-88, Zitat S. 86.

30 R. Zippelius weist in dem mir erst nachträglich bekanntgewordenen Beitrag ,Legitimation im demokratischen Verfassungsstaat" (in: A. Achterberg/W. Krawietz (Hrsg.), Legitimation des modernen Staates, Wiesbaden 1981, S. 84-94) darauf hin, daß die institutionellen Vorkehrungen des Rechtsstaats ,keineswegs auf eine bloße ,Legitimation durch Verfahren' hinaus(laufen): Gefordert sind vielmehr prozedurale Vorkehrungen im Dienste der größtmöglichen Annäherung an ein Ergebnis, das für möglichst viele seinem Inhalt nach konsensf ähig ist, d.h. an ein Ergebnis, das vor dem Gewissen -- und speziell in Gerechtigkeitsfragen vor dem Rechtsgefühl - möglichst vieler bestehen kann" (a.a.O., S. 90). Die vorliegenden Darlegungen sollen nicht diese Norm in Frage stellen, jedoch darauf hinweisen, daß die tatsächlichen Verhältnisse sich in eine Richtung entwickeln, die es der Rechtspolitik immer mehr erschwert, ihr getecht zu werden. Inwieweit Normkonformität noch auf Konsens und inwiefern auf bloßer Ftigsamkeit infolge der Furcht von Nachteilen beruht, und welchen Folgen von einer solchen Verschiebung zu erwarten sind, dies scheinen mir die entscheidenden Fragen in unserem Zusammenhang.

31 Dies postuliert M. Kriele, Recht und praktische Vernunft, Göttingen 1979.

32 F.X. Kaufmann, Sicherheit, a.a.O., (FN 9), S. 75 ff. 
33 Vgl. hierzu P. Noll, Gründe für die soziale Unwirksamkeit von Gesetzen. In: JB für Rechtssoziologie und Rechtstheorie Bd. 3, a.a.O., (FN 1), S. 259-269.

34 M. Rümelin, Die Rechtssicherheit, Tübingen 1924, S. 7.

$35 \mathrm{Vgl}$. H. Döring/F.-X. Kaufmann, Kontingenzerfahrung und Sinnfrage. In: Christlicher Glaube in moderner Gesellschaft. Teilbd. 9, Freiburg i.Br., 1981, S. 8 ff.

$36 \mathrm{Vgl}$. hierzu K. Gretschmann, Wirtschaft im Schatten von Markt und Staat. Grenzen und Möglichkeiten seiner Alternativökonomie. Frankfurt/M. 1983.

$37 \mathrm{Vgl}$. P. A. Berger/B. Berger/H. Kellner, Das Unbehagen in der Modernität. Frankfurt/New York 1973, bes. S. $42 \mathrm{ff}$.

38 Für erste Oberlegungen in diesem Zusammenhang vgl. R. Wahl, Die bürokratischen Kosten des Rechts- und Sozialstaats. In: Die Verwaltung 13 (1980) S. 273-296. - K. Eckel, Das Sozialexperiment. Finales Recht als Bindeglied zwischen Politik und Sozialwissenschaft. Zeitschrift für Soziologie 7 (1978) S. 39-55. - W. Hugger, Legislative Effektivitätssteigerung: von den Grenzen der Gesetzesevaluierbarkeit zum Gesetz auf Zeit. In: Politische Vierteljahresschrift XX (1979) S. 202-220.

\section{Diskussion des Referats Kaufmann \\ Leitung: R. Lautmann}

Kaufmann eröffnete die Aussprache, indem er den Begriff des „Rechtsgefühls“ als wichtigen Bestandteil des juristischen Diskurses kennzeichnete und gleichzeitig betonte, daß eine soziologische Untersuchung dieses Konzepts wesentlich sei für seine inhaltliche Präzisierung. So gesehen könne man das Rechtsgefühl als ein Mittelglied zwischen der Rechtssetzung und der Dynamik sozialer Vorgänge verstehen. Es diene als eine Art von Mechanismus zur Normkonformität, d. h. es fördere die Akzeptanz von Rechtsnormen durch die Bürger und vermindere das Potential möglicher sozialer Konflikte. Wo Rechtsgefühle nur mehr rudimentär vorhanden seien, da entstünden Legitimationsprobleme. Generell gesehen besitze das Konzept des Rechtsgefühls eine große demokratietheoretische Relevanz. Der demokratische Rechtsstaat stelle im Grundsatz zwar die Verbindung zwischen Recht und Rechtsgefühl her, doch seien in ihm Tendenzen wirksam, die auf eine Unterhöhlung dieser Verbindung hinausliefen. Das mache sich u. a. daran bemerkbar, daß heutzutage eine zunehmende Unsicherheit in der Öffentlichkeit wie bei den Juristen selbst hinsichtlich der Frage herrsche, was dieses „Rechtsgefühl“ denn nun eigentlich sei. Nun vermöge ein Rechtsgefühl sich nur aus dem Kontakt mit der Rechtsordnung im Medium des alltäglichen Lebens zu entwickeln. Dabei gelte, daß die Ausdrucksformen des Rechtsgefühls mit wachsender Komplexität der sozialen Bedingungen immer unbestimmter würden. Man denke hier nur an das Absterben lokaler Rechtstraditionen in der modernen Gesellschaft und an das damit gegebene Aufhören der früher vorhandenen Möglichkeit, auf ältere, ,sedimentäre“ Formen des Rechts zurückzugreifen. Hier sei das riesige Wachstum der Verwaltungstätigkeit von entscheidender Bedeutung geworden. Mit ihm sei aus dem Recht, das vordem der Friedenswahrung zwischen den Bürgern gedient habe, ein bürokratischer Steuerungsmechanismus geworden. Die vielbeklagte „Inflation des Rechts“ sei eine Folge dieses Vorgangs. Die heutige Rechtserfahrung des Bürgers sei wesentlich Verwaltungserfahrung und nicht mehr vorrangig Erfahrung mit Gerichten. Man könne hier fast von einer Art „Entfremdung" sprechen, die mit dem Unbestimmtwerden des Rechtsgefühls in 